\title{
Role of Image-Enhanced Endoscopy in Pancreatobiliary Diseases
}

\author{
Yun Nah Lee, Jong Ho Moon and Hyun Jong Choi \\ Digestive Disease Center and Research Institute, Department of Internal Medicine, Soonchunhyang University College of Medicine, Bucheon, \\ Korea
}

Recent advances in cholangiopancreatoscopy technology permit image-enhanced endoscopy (IEE) for pancreatobiliary diseases. There are limitations in endoscopy performance and in the study of the clinical role of IEE in bile duct or pancreatic duct diseases. However, currently available IEEs during cholangiopancreatoscopy including traditional dye-aided chromoendoscopy, autofluorescence imaging, narrow-band imaging, and i-Scan have been evaluated and reported previously. Although the clinical role of IEE in pancreatobiliary diseases should be verified in future studies, IEE is a useful promising tool in the evaluation of bile duct or pancreatic duct mucosal lesions. Clin Endosc 2018;51:541-546

Key Words: Biliary tract diseases; Endoscopy; Digestive system; Narrow-band imaging

\section{INTRODUCTION}

In contrast to gastrointestinal tract diseases, endoscopic direct visual evaluation with tissue acquisition is not the mainstay for the diagnostic approach to pancreatobiliary diseases. Conventional cross-sectional imaging modalities including transabdominal ultrasonography, computed tomography, magnetic resonance imaging with cholangiopancreatography, endoscopic ultrasonography, and endoscopic retrograde cholangiopancreatography (ERCP) are primary and principal options for the evaluation of pancreatobiliary diseases until now. Endoscopic visual evaluation of the bile duct or pancreatic duct is limited fundamentally by anatomical features and demands specialized equipment and experienced endoscopists. Recently, however, there have been remarkable advancements in cholangiopancreatoscopy systems. These advances have

Received: October 30, 2018 Revised: November 15, 2018 Accepted: November 16, 2018

Correspondence: Jong Ho Moon

Digestive Disease Center and Research Institute, Department of Internal Medicine, Soonchunhyang University Bucheon Hospital, Soonchunhyang University College of Medicine, 170 Jomaru-ro, Wonmi-gu, Bucheon 14584, Korea

Tel: +82-32-621-5094, Fax: +82-32-621-5080, E-mail: jhmoon@schmc.ac.kr ORCID: https://orcid.org/0000-0002-3946-9944

cc This is an Open Access article distributed under the terms of the Creative Commons Attribution Non-Commercial License (http://creativecommons.org/ licenses/by-nc/3.0) which permits unrestricted non-commercial use, distribution, and reproduction in any medium, provided the original work is properly cited. led to the spread of the performance of cholangioscopy or pancreatoscopy that had previously been limited to special academic centers. ${ }^{1}$

Endoscopic direct visual assessment of the mucosa in the bile duct and pancreatic duct has clinical value in the diagnosis and treatment of pancreatobiliary diseases. Image-enhanced endoscopy (IEE) may provide clearer and more enhanced images than conventional white light imaging. ${ }^{2}$ However, performing and studying IEE with cholangiopancreatoscopy is more limited than with gastrointestinal endoscopy. Despite the advances in cholangioscopic and pancreatoscopic systems, there remain limitations in performing the procedure. In addition, IEE is not available in some modalities of cholangiopancreatoscopic systems. Furthermore, characteristic endoscopic findings of white light imaging in cholangiopancreatoscopy have not yet been fully evaluated and established. The clinical role of cholangioscopy and tissue acquisition without IEE has now been studied and reported extensively. ${ }^{3,4}$ The study and data about pancreatoscopic findings are more deficient than those of cholangioscopy. Nevertheless, it is expected that IEE in cholangiopancreatoscopy may likely be a valuable tool in evaluating pancreatobiliary diseases. ${ }^{5}$ Continuous progress is being made with IEE in cholangiopancreatoscopy along with the ongoing advances of endoscopy technology. In this review, we briefly present the recent advances in cholangiopancreatoscopic systems, available IEE in cholangiopancreatoscopy 
systems, and current status of IEE for the evaluation of pancreatobiliary diseases.

\section{CHOLANGIOPANCREATOSCOPY SYSTEMS}

Peroral cholangioscopy (POCS) and pancreatoscopy (POPS) have been developed in three different systems: (1) traditional mother-baby endoscope system (dual-operator system), (2) single-operator "mother-baby" endoscope system (SplyGlass Direct Visualization System; Boston Scientific, Natick, MA, USA), and (3) direct POCS using an ultraslim endoscope (single-operator and single-endoscope system). The mother-baby endoscope system had been the mainstay in POCS preceding the other two systems. The main disadvantages include fragility of a choledochoscope ("baby scope"), small working channel, and difficult maneuverability in the close cooperation of two skilled endoscopists. ${ }^{1,6}$ Recently developed video choledochoscopes and pancreatoscopes provide clear images with improved resolution and available IEE, which is narrow-band imaging (NBI)..$^{7-9}$ The SpyGlass system does not provide an IEE mode equipped in the system. A recently developed new version, the SpyGlass DS system, presents improved digital images, and it is expected that IEE will be available in the near future. Direct POCS using slim endoscopes allows the acquisition of clear images in high resolution and performing available IEE modes according to each endoscopy company, such as NBI, autofluorescence imaging (AFI), i-Scan, or Flexible Spectral Imaging Color Enhancement (FICE). Direct POCS is currently the most economic POCS modality that uses a conventional endoscopy system. However, it requires learning time and assisting devices, such as a balloon catheter for successful advancement of the slim endoscope into the bile duct. In addition, direct POCS or POPS can only be performed in patients with a dilated duct, and it is occasionally difficult to sustain a stable scope position. ${ }^{10,11}$

Percutaneous transhepatic cholangioscopy (PTCS) is a traditional cholangioscopy that has been widely used. PTCS has good maneuverability since the length of choledochoscope is short. Recently developed video choledochoscopes provide better endoscopic images compared to conventional fiberscopes and are also equipped with IEE function. However, it usually takes two or three weeks for a transhepatic tract to form prior to carrying out PTCS and causes discomfort to patients because of external drainage. In addition, rare significant complications and tract-related cancer spreading in malignant diseases can develop. Despite some disadvantages, PTCS has its own indispensable diagnostic and therapeutic roles in patients with bile duct diseases. ${ }^{12}$ Hamada et al. re- ported performing PTCS with an ultraslim video endoscope after tract dilation up to $16 \mathrm{~F}^{13}$ PTCS using a slim endoscope can provide good images in high resolution with various IEE modes like those of direct POCS.

\section{TRADITIONAL CHROMOENDOSCOPY}

Traditional chromoendoscopy using dye solutions in the bile duct has been demonstrated in several studies. Maetani et al. evaluated methylene blue staining in the bile duct through PTCS and reported that the cancerous epithelia were significantly less stained than the normal or metaplastic epithelia. ${ }^{14}$ Hoffman et al. evaluated methylene-blue-aided chromoendoscopy through mother-baby POCS. ${ }^{15}$ Whereas normal/ non-dysplastic mucosa was characterized by homogeneous staining patterns, inhomogeneous dark staining was observed in inflamed and dysplastic areas. Chromoendoscopy in the bile duct highlighted mucosal lesions of interest during POCS and permitted better characterization. ${ }^{15}$ However, chromoendoscopy by methylene blue in the bile duct is affected by mucus or exudate that is also stained dark. ${ }^{16}$ Since the development of video cholangioscopes, IEE during cholangioscopy or POPS can now be performed by virtual chromoendoscopy such as NBI or AFI.

\section{AUTOFLUORESCENCE IMAGING}

Normal mucosa appears green, but neoplastic lesions appear dark green or black on AFI. Itoi et al. evaluated the diagnostic yield of video PTCS with AFI. ${ }^{16}$ Interestingly, AFI detected lateral cancer spread lesions that were missed by white light imaging in some patients. However, the frequency of false-positives increased on AFI in comparison with white light imaging. The inferior specificity of AFI might be affected by bile or non-neoplastic granular mucosa appearing as a dark green color. ${ }^{6,16}$

\section{NARROW-BAND IMAGING}

NBI permits better characterization of mucosal surface structures and superficial vessels in the bile duct or pancreatic duct than conventional white light imaging (Fig. 1). ${ }^{7.8}$ Osanai et al. reported a prospective study of video POCS (motherbaby system) in evaluating indeterminate bile duct lesions and preoperative mucosal cancerous extension. ${ }^{17}$ POCS with NBI distinguished benign from malignant lesions in $92.1 \%$ of 87 patients. The accuracy for the mucosal cancer extension by 
ERCP alone and ERCP plus POCS with NBI were $73.5 \%$ and $83.7 \%$, respectively. ${ }^{17}$ Although the diagnostic yield of NBI in comparison with white light imaging alone was not evaluated, NBI was useful in differentiating benign from malignant lesions by enhancing the surface structure and margins of the lesions in 34 of 38 patients (92.1\% accuracy; $96.4 \%$ sensitivity; $80.0 \%$ specificity). The surface structure and margin of the mucosal cancer extension were enhanced in $95.6 \%$ of patients by NBI. Nishikawa et al. presented a prospective study of video POCS for preoperative assessment of longitudinal extension of cholangiocarcinoma. ${ }^{18}$ However, the authors reported that the diagnostic accuracy of POCS for longitudinal extension of cancer was not improved by applying NBI, although NBI actually facilitated the identification of mucosal changes. ${ }^{18}$ Azeem et al. evaluated the role of NBI in identifying dysplasia in patients with primary sclerosing cholangitis. ${ }^{19}$ However, NBI-directed biopsies did not improve the dysplasia detection rate. ${ }^{19}$ The authors reported that NBI was useful in
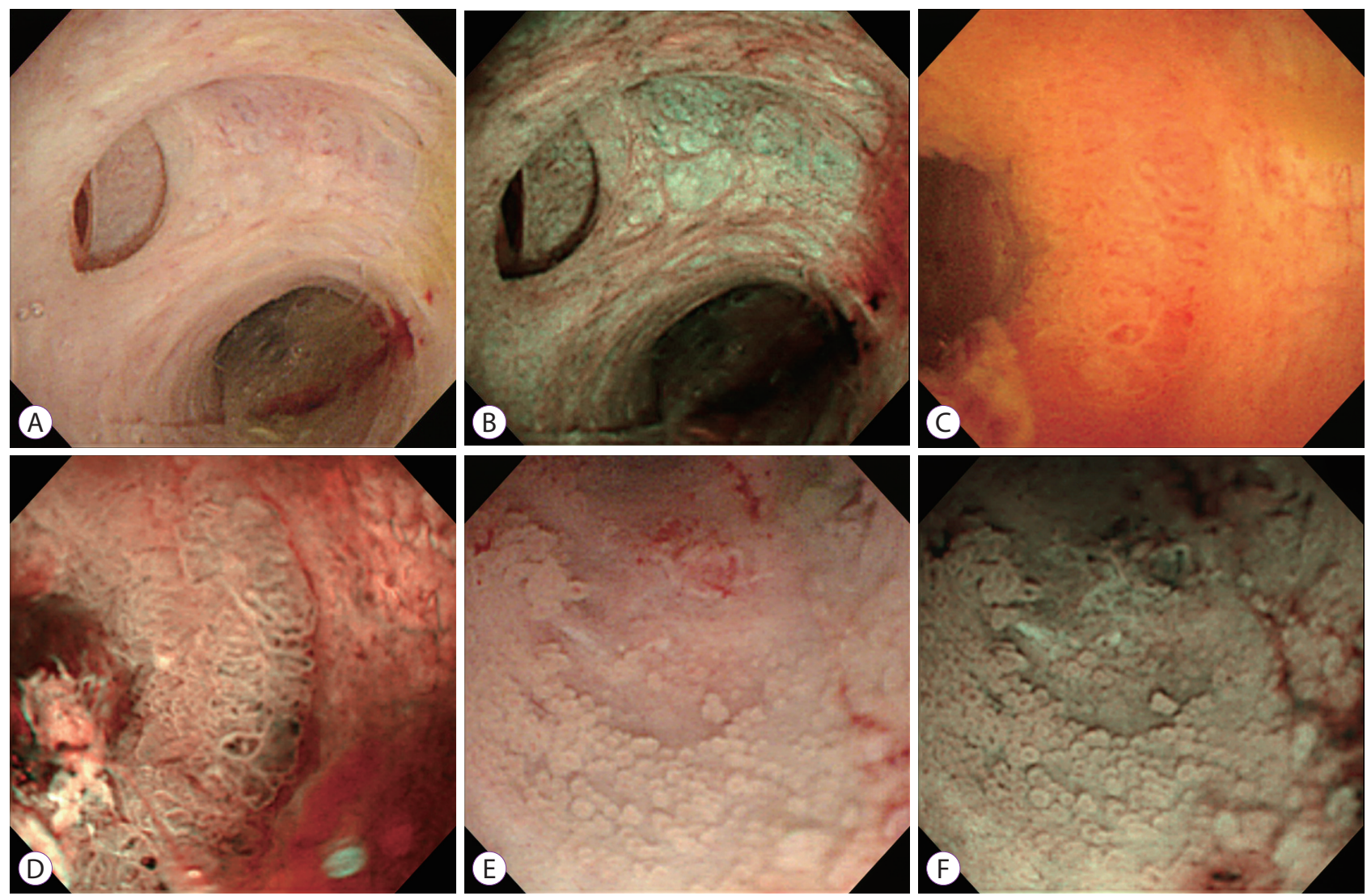

Fig. 1. Peroral cholangioscopy with narrow-band imaging (NBI). White light image $(A)$ and $N B I(B)$ of the normal bile duct. White light image $(C$ and $E)$ and $N B I(D$ and $F)$ of intraductal papillary neoplasm in the bile duct.
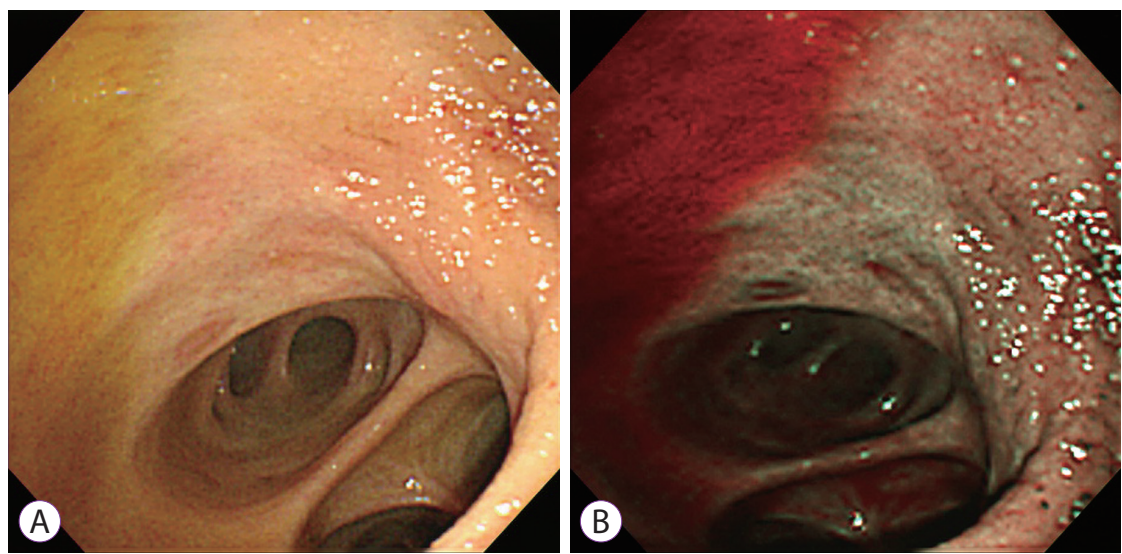

Fig. 2. Peroral cholangioscopy with narrow-band imaging (NBI). Bile appears as red-colored fluid in NBI. 
delineating tumor margins in two of four patients with cholangiocarcinoma. The usefulness of NBI during cholangioscopy has been demonstrated in several pilot studies with small numbers of patients.

One of the limitations of NBI in the bile duct is that the bile appears in a dark red color (Fig. 2). In addition, currently used cholangioscopy systems do not have optical magnifying function, while NBI in the gastrointestinal tract has generally been used with a magnifying endoscope. Magnifying endoscopy using NBI can provide clearer images than those provided by currently available cholangioscopes. ${ }^{20}$ Ishida et al. present- ed an ex vivo study of magnifying endoscopy with NBI in surgically resected common bile duct mucosa. ${ }^{21}$ The authors compared the magnifying endoscopy with NBI findings to the histopathology. ${ }^{21}$ They reported that NBI provided clearer visualization of both microvessels and mucosal structures in comparison with that provided by white light imaging during magnifying endoscopy. Interestingly, although magnifying endoscopy with NBI provided the best endoscopic visualization of the bile duct mucosa, it was difficult to distinguish between neoplastic and non-neoplastic mucosa with severe inflammation. These results may point toward the fundamen-

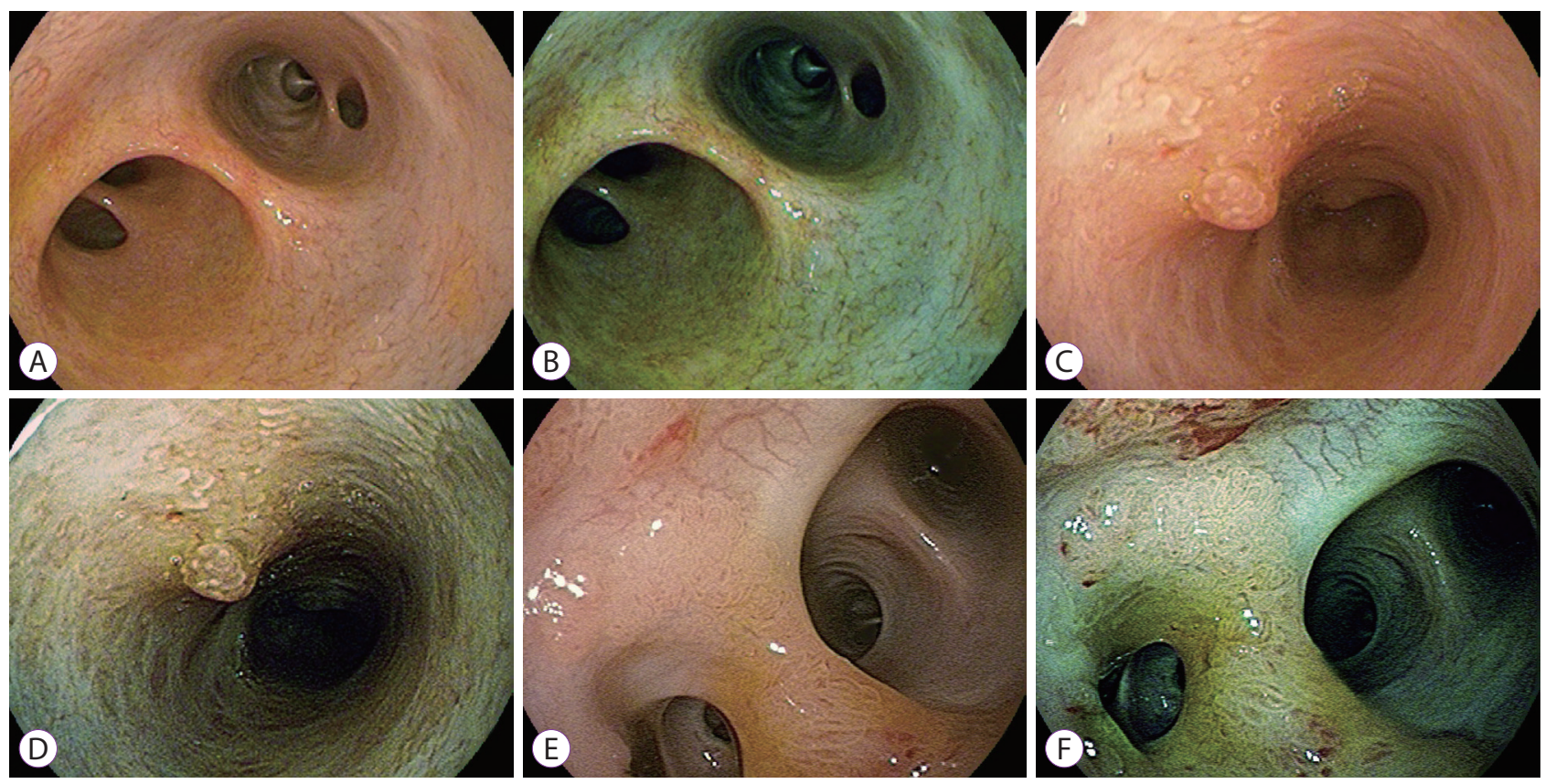

Fig. 3. Peroral cholangioscopy with i-Scan. White light image (A) and i-Scan image (B) of normal bile duct at the hilar bifurcation. White light image (C) and i-Scan image (D) of an inflammatory polyp. White light image $(E)$ and i-Scan image $(F)$ of an intraductal papillary neoplasm in the bile duct.
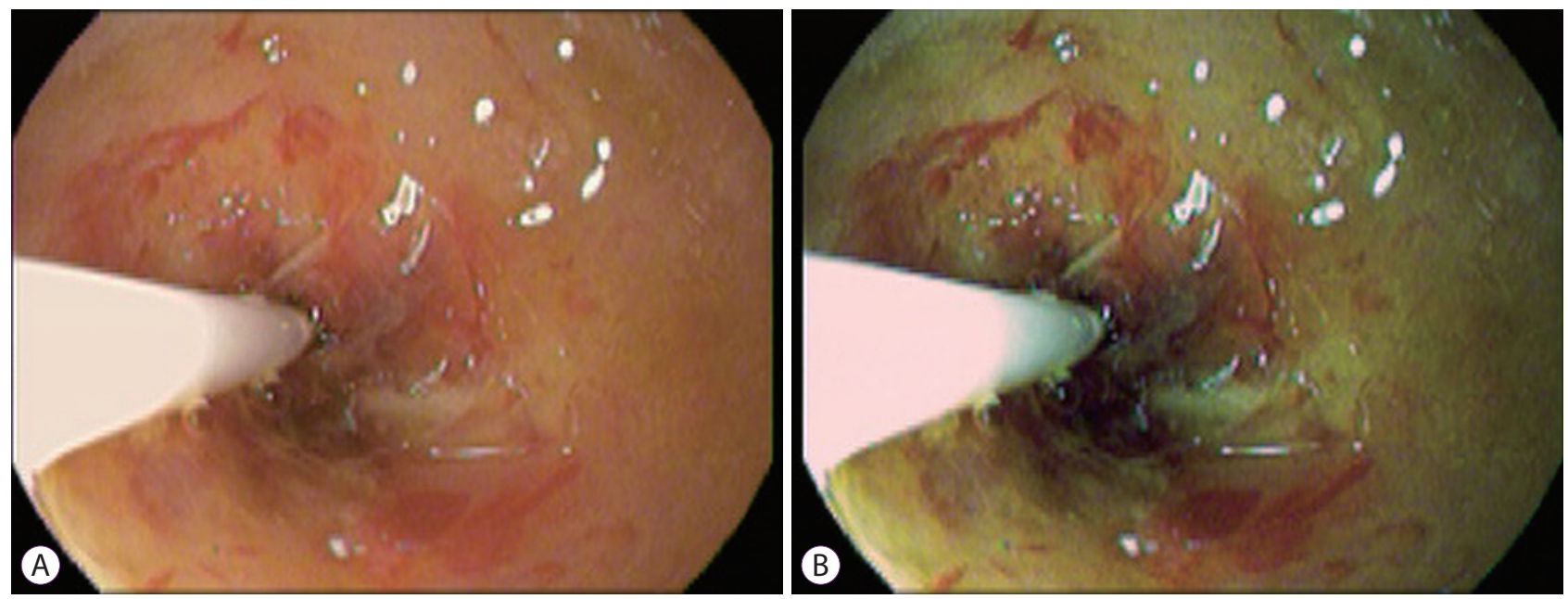

Fig. 4. Twin mode of i-Scan. 
tal limitation of endoscopic evaluation with IEE for bile duct lesions and emphasize the importance of pathologic confirmation by tissue acquisition.

\section{i-Scan}

$\mathrm{i}$-Scan is another image enhancement system through computed, dynamic, digital image processing. i-Scan provides three different enhancement options that highlight specific anatomical features (Fig. 3). White light and i-Scan imaging can be displayed in twin mode simultaneously side by side (Fig. 4). Lee et al. evaluated the diagnostic yield of i-Scan compared with conventional white light imaging for bile duct lesions through direct POCS using an ultraslim endoscope. ${ }^{4}$ The quality of endoscopic visualization with i-Scan was significantly higher than that with conventional white light imaging during direct POCS for surface structure $(p=0.04)$, surface microvascular architecture $(p=0.01)$, and margins $(p=0.02)$. The overall diagnostic accuracies of the visual diagnosis of white light imaging and i-Scan were $75 \%$ and $90 \%$, respectively $(p=0.20) .{ }^{4}$ In contrast to NBI, i-Scan is not interfered by bile. There are no other studies of i-Scan in cholangiopancreatoscopy. Additional studies are needed to evaluate the role of digital virtual chromoendoscopy such as i-Scan or FICE during cholangiopancreatoscopy.

\section{LIMITATIONS OF IMAGE-ENHANCED ENDOSCOPY IN PANREATOBILIARY DISEASES}

Besides hurdles in the endoscopy technology and performance of cholangioscopy or pancreatoscopy, one of the inherent limitations is the nature of cholangiocarcinoma, i.e., submucosal tumor spreading. Direct endoscopic visualization of neoplastic mucosal lesions using cholangioscopy is rightly helpful to evaluate and diagnose the lesions. Cholangioscopy with IEE like NBI is very useful in evaluating patients with intraductal papillary neoplasms of the bile duct that have characteristic mucosal changes and features. ${ }^{22,23}$ Minimal mucosal lesions that are missed on conventional cross-sectional imaging and ERCP can be incidently detected by cholangioscopic evaluation, and IEE may improve the detection rate. However, there is a fundamental limitation in endoscopic evaluation for submucosal-spreading tumor lesions.

Characteristic endoscopic findings of neoplastic or non-neoplastic mucosal lesions have been described in previous reports. ${ }^{6,24}$ Although descriptions and definitions of endoscopic findings are mostly agreed and consented by en- doscopists, the findings have not yet been fully evaluated and established. There are not enough studies and data to achieve establishment yet. Tortuous enlarged mucosal vessels indicating "tumor vessels" may appear in severe inflammation, especially in patients with sclerosing cholangitis. ${ }^{25}$ Distinguishing neoplastic lesions from non-neoplastic lesions may be difficult in severe inflammatory mucosa.

\section{CONCLUSIONS}

Recent advances in cholangiopancreatoscopy technology have allowed the expansion of the clinical role of direct endoscopic evaluation in pancreatobiliary diseases. Currently available IEE performed with cholangioscopy or pancreatoscopy provides enhanced endoscopic images by each specific technology and may have additional clinical roles. There are technical challenges to be overcome and fundamental limitations in IEE on cholangiopancreatoscopy for pancreatobiliary diseases. Nevertheless, IEE in cholangioscopy or pancreatoscopy should be verified in future studies as a useful promising tool for diagnosing pancreatobiliary diseases.

\section{Conflicts of Interest}

The authors have no financial conflicts of interest.

\section{REFERENCES}

1. Moon JH, Terheggen G, Choi HJ, Neuhaus H. Peroral cholangioscopy: diagnostic and therapeutic applications. Gastroenterology 2013;144:276282.

2. Kim WJ, Park SY, Park I, et al. Increased detection of colorectal polyps in screening colonoscopy using high definition i-Scan compared with standard white light. Clin Endosc 2016;49:69-75.

3. Sun X, Zhou Z, Tian J, et al. Is single-operator peroral cholangioscopy a useful tool for the diagnosis of indeterminate biliary lesion? A systematic review and meta-analysis. Gastrointest Endosc 2015;82:79-87.

4. Lee YN, Moon JH, Choi HJ, et al. Direct peroral cholangioscopy for diagnosis of bile duct lesions using an i-Scan ultraslim endoscope: a pilot study. Endoscopy 2017;49:675-681.

5. Mounzer R, Austin GL, Wani S, Brauer BC, Fukami N, Shah RJ. Per-oral video cholangiopancreatoscopy with narrow-band imaging for the evaluation of indeterminate pancreaticobiliary disease. Gastrointest Endosc 2017;85:509-517.

6. Ishida Y, Itoi T, Okabe Y. Can image-enhanced cholangioscopy distinguish benign from malignant lesions in the biliary duct? Best Pract Res Clin Gastroenterol 2015;29:611-625.

7. Itoi $\mathrm{T}$, Sofuni $\mathrm{A}$, Itokawa $\mathrm{F}$, et al. Initial experience of peroral pancreatoscopy combined with narrow-band imaging in the diagnosis of intraductal papillary mucinous neoplasms of the pancreas (with videos). Gastrointest Endosc 2007;66:793-797.

8. Itoi T, Sofuni A, Itokawa F, et al. Peroral cholangioscopic diagnosis of biliary-tract diseases by using narrow-band imaging (with videos). Gastrointest Endosc 2007;66:730-736.

9. Parsi MA, Jang S, Sanaka M, Stevens T, Vargo JJ. Diagnostic and thera- 
peutic cholangiopancreatoscopy: performance of a new digital cholangioscope. Gastrointest Endosc 2014;79:936-942.

10. Ishida Y, Itoi T, Okabe Y. Types of peroral cholangioscopy: how to choose the most suitable type of cholangioscopy. Curr Treat Options Gastroenterol 2016;14:210-219.

11. ASGE Technology Committee, Komanduri S, Thosani N, et al. Cholangiopancreatoscopy. Gastrointest Endosc 2016;84:209-221.

12. Choi JH, Lee SK. Percutaneous transhepatic cholangioscopy: does its role still exist? Clin Endosc 2013;46:529-536.

13. Hamada T, Tsujino T, Sasahira N, et al. Percutaneous transhepatic cholangioscopy with an ultraslim video upper endoscope with $\mathrm{CO}_{2}$ insufflation: a feasibility study. Gastrointest Endosc 2011;74:696-699.

14. Maetani I, Ogawa S, Sato M, Igarashi Y, Sakai Y, Shibuya K. Lack of methylene blue staining in superficial epithelia as a possible marker for superficial lateral spread of bile duct cancer. Diagn Ther Endosc 1996;3:29-34.

15. Hoffman A, Kiesslich R, Bittinger F, Galle PR, Neurath MF. Methylene blue-aided cholangioscopy in patients with biliary strictures: feasibility and outcome analysis. Endoscopy 2008;40:563-571.

16. Itoi T, Shinohara Y, Takeda K, Nakamura K, Takei K. Improvement of choledochoscopy: chromoendocholedochoscopy, autofluorescence imaging, or narrow-band imaging. Dig Endosc 2007;19(Suppl 1):S95-S104.

17. Osanai M, Itoi T, Igarashi Y, et al. Peroral video cholangioscopy to evaluate indeterminate bile duct lesions and preoperative mucosal cancerous extension: a prospective multicenter study. Endoscopy 2013;45:635-642.

18. Nishikawa T, Tsuyuguchi T, Sakai Y, et al. Preoperative assessment of longitudinal extension of cholangiocarcinoma with peroral video-cholangioscopy: a prospective study. Dig Endosc 2014;26:450-457.

19. Azeem N, Gostout CJ, Knipschield M, Baron TH. Cholangioscopy with narrow-band imaging in patients with primary sclerosing cholangitis undergoing ERCP. Gastrointest Endosc 2014;79:773-779.e2.

20. Ishida Y, Okabe Y, Kaji R, et al. Evaluation of magnifying endoscopy using narrow band imaging using ex vivo bile duct (with video). Dig Endosc 2013;25:322-328.

21. Ishida $\mathrm{Y}$, Okabe $\mathrm{Y}$, Yasumoto $\mathrm{M}$, et al. Ex vivo magnifying endoscopic observation of bile duct mucosa using narrowband imaging. J Hepatobiliary Pancreat Sci 2018;25:433-439.

22. Brauer BC, Fukami N, Chen YK. Direct cholangioscopy with narrow-band imaging, chromoendoscopy, and argon plasma coagulation of intraductal papillary mucinous neoplasm of the bile duct (with videos). Gastrointest Endosc 2008;67:574-576.

23. Itoi T, Sofuni A, Itokawa F, Tsuchiya T, Kurihara T. Evaluation of peroral videocholangioscopy using narrow-band imaging for diagnosis of intraductal papillary neoplasm of the bile duct. Dig Endosc 2009;21 Suppl 1: S103-S107.

24. Itoi T, Neuhaus $\mathrm{H}$, Chen YK. Diagnostic value of image-enhanced video cholangiopancreatoscopy. Gastrointest Endosc Clin N Am 2009;19:557566.

25. Itoi T, Kamisawa T, Igarashi Y, et al. The role of peroral video cholangioscopy in patients with IgG4-related sclerosing cholangitis. J Gastroenterol 2013;48:504-514. 